Review / Meta-analyses

\title{
Electroretinography in psychiatry: A systematic literature review
}

\author{
Peter Youssef ${ }^{\mathrm{a}}$, Siddharth Nath ${ }^{\mathrm{b}}$, Gary A Chaimowitz ${ }^{\mathrm{c}, \mathrm{d}}$, Sebastien S. Prat ${ }^{\mathrm{c}, \mathrm{d}, *}$ \\ a Bachelor of Health Sciences Program, McMaster University, Hamilton, Ontario, Canada \\ ${ }^{\mathrm{b}} \mathrm{MD} / \mathrm{PhD}$ Program, McMaster University, Hamilton, Ontario, Canada \\ ${ }^{\mathrm{c}}$ Department of Psychiatry and Behavioural Neurosciences, McMaster University, Hamilton, Ontario, Canada \\ d Forensic Psychiatry Program, St. Joseph's Healthcare Hamilton, Hamilton, Ontario, Canada
}

\section{A R T I C L E I N F O}

\section{Article history:}

Received 7 September 2019

Accepted 7 September 2019

Available online 22 September 2019

\section{Keywords:}

Electroretinography

Electroretinogram

Psychiatry

Diagnostic tools

\begin{abstract}
A B S T R A C T
This review aims to consolidate the available information on use of electroretinography as a diagnostic tool in psychiatry. The electroretinogram (ERG) has been found to have diagnostic utility in cocaine withdrawal (reduced light-adapted b-wave response), major depressive disorder (reduced contrast gain in pattern ERG), and schizophrenia (reduced a- and b-wave amplitudes). This review examines these findings as well as the applicability of ERG to substance use disorder, Alzheimer's disease, autism spectrum disorder, panic disorder, eating disorders, attention deficit hyperactivity disorder, and medication use. While there have been promising results, current research suffers from a lack of specificity. Further research that quantifies anomalies in ERG present in psychiatric illness is needed.
\end{abstract}

(C) 2019 Elsevier Masson SAS. All rights reserved.

\section{Introduction}

Diagnosis of mental illness is often a lengthy and involved process. As definitions and categorizations of diseases become increasingly complex, a search for objective biomarkers of mental illness is underway to facilitate swift and accurate clinical assessment [1]. Because the retina uses neurotransmitters for phototransduction, it is hypothesized that the deregulation of neurotransmitter physiology present in many mental illnesses may be detectable through assessment of retinal function.

The electroretinogram (ERG), is a non-invasive diagnostic test that measures electrical activity generated by neuronal and nonneuronal cells within the retina and has shown utility in the assessment of psychiatric illnesses [1-3]. It measures electrical impulses through a contact lens which can detect summation of retinal electrical activity at the corneal surface. In mouse models, it has been shown that alterations in retinal dopaminergic and serotonergic neurotransmission parallel abnormalities observed in ERG recordings [2].

Abbreviations: ADHD, attention deficit hyperactivity disorder; $\mathrm{CI}$, confidence interval; ERG, electroretinogram/electroretinography; fERG, flash electroretinogram/electroretinography; ISCEV, international society for clinical electrophysiology of vision; MDD, major depressive disorder; mfERG, multifocal electroretinography/electroretinogram; ms, millisecond; pERG, pattern electroretinogram/electroretinography; PRISMA, preferred reporting items for systematic reviews and meta-analyses.

* Corresponding author at: Forensic Psychiatry Program, St. Joseph's Healthcare Hamilton, Hamilton, ON L8N 3K7, Canada.

E-mail address: prats@mcmaster.ca (S.S. Prat).
The ERG outputs multiple waveforms, which represent different components of retinal electrophysiology [4]. Of these, two waveforms have been shown to be especially important in the setting of psychiatry: the a-wave and the b-wave. The a-wave is representative of photoreceptor function and is measured as the height of the baseline electrical activity before flash onset to the trough of the first wave. The b-wave reflects bipolar cell function and is measured from the trough of the a-wave to the peak of the second wave [4]. Examples of ERG waveforms are shown in Fig. 1.

There exist different types of ERGs, which can assess varied components of the retina. The full-field flash ERG (fERG) assesses the electrophysiological response to a flash of light and is wellestablished and routinely used in ophthalmology [4]. It is also under investigation for applications in psychiatry. The fERG assesses retinal function under dark-adapted (scotopic) or lightadapted (photopic) settings. The scotopic fERG is usually indicative of rod function, while the photopic fERG commonly correlates with cone function [4]. Mixed cone/rod responses may also be elicited on scotopic ERG. In addition to fERG, pattern ERG (PERG) is also under evaluation in psychiatric populations. PERG provides information regarding macular, bipolar, and retinal ganglion cell function by stimulation with a rapidly reversing high-contrast black and white checkerboard or alternating horizontal and vertical lines, and therefore also allows for inferences of retinal contrast sensitivity [7,8]. Moreover, some investigators have also begun to study the newer multifocal ERG ( $\mathrm{mfERG}$ ) in the setting of mental illness. This ERG measures the response from hundreds of points on the retina simultaneously and creates a 'topographic map' of retinal functioning [9]. 


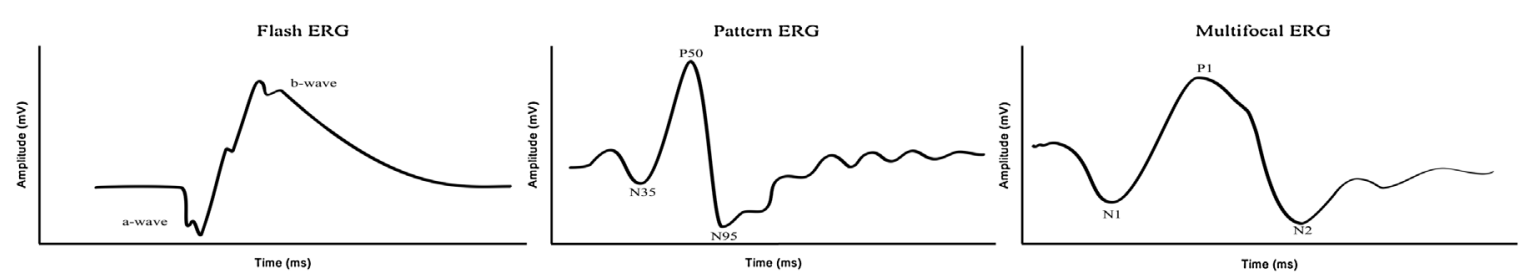

Fig. 1. fERG, PERG, and mfERG waveforms and parameters. Waveforms based on information provided in included studies.

Although there remains contention with regards to the neurochemical basis of ERG waves, retinal physiology is known to be primarily dependent on glutamate [7]. Thus, it is hypothesized that changes to the glutamate neurotransmitter system, as are observed in many psychiatric illnesses, may manifest in different ERG profiles in varied disease states. Some groups hypothesize that the retinal endocannabinoid system, dopamine system, or the GABAergic system may also be at play, allowing for insight into psychiatric conditions resulting from aberrant functioning of these signaling pathways $[2,5,6]$.

This review explores variations in ERG waveforms across several different psychiatric populations and highlights potential applications for ERG in the detection and assessment of mental illness.

\section{Material and methods}

\subsection{General methods}

The present review was conducted in line with the Preferred Reporting Items for Systematic Reviews and Meta-Analyses (PRISMA) statement [10].

\subsection{Literature search}

We performed a systematic search of the Web of Science electronic database using the following keywords: 'electroretinogram', 'electroretinography', and ['electroretinography' + psychiatry (topic)]. To ensure we captured all available evidence in the

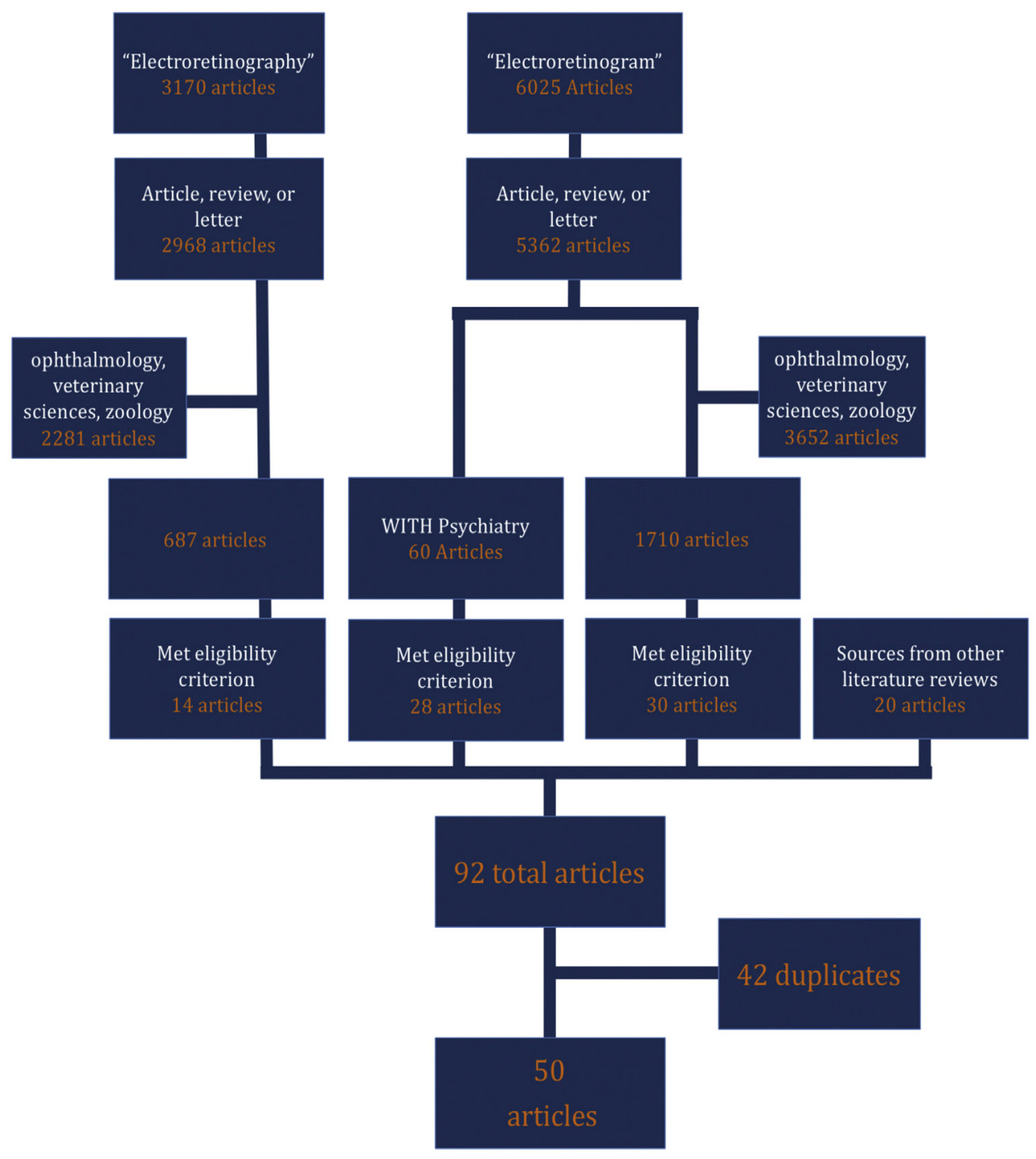

Fig. 2. PRISMA flow diagram. The selection process for identifying eligible studies is shown. 
field, our sole eligibility criteria was that ERG was used on human subjects for diagnosis or assessment of mental illness. We excluded articles not investigating psychiatric conditions, articles with the keyword 'ophthalmology', as well as wet lab research and animal studies. Our electronic search was supplemented by reviewing the references of included articles. For the purposes of our review, Alzheimer's disease was considered a psychiatric condition and was thus included, while epilepsy was considered a neurological condition and was excluded. We included both full reports and conference proceedings, provided that a subsequent article had not been published.

\subsection{Data collection}

We downloaded the complete articles of all included studies. We created data extraction forms with fields for: population studied, type of ERG (fERG, PERG, mfERG), study design, and ERG results.

\section{Results}

\subsection{General results}

Our literature search yielded 9,195 results, from which 55 articles met our inclusion criteria (Fig. 2). Articles covered a spectrum of psychiatric illnesses including substance use, depressive disorders, schizophrenia, panic disorder, and eating disorders. Most articles focused on the applicability of ERG to substance use, depressive disorders, and schizophrenia, with a minority investigating panic disorder and eating disorders. Distribution of psychiatric illnesses amongst the articles is shown in Fig. 3.

Included articles covered over 30 years of research and a timeline of how the landscape of the field has evolved is shown in Fig. 4. Notably, there has been increased interest in the applicability of ERG to psychiatric illness. Use of ERG as a diagnostic tool has had an early start in the scientific literature, however, there has been little success in establishing it for clinical use. As shown, there are also periods with little research progress, concordant with periods of plateauing in technological innovation.

\subsection{Substance use}

We found 15 articles evaluating the effects of various substances on ERG [6,11-24]. Of these, nine studied the effects of cocaine, three studied cannabis, two studied alcohol, and one studied mixed drug use. A complete summary of the included studies and their findings is shown in Table 1.

In the setting of cocaine use, it was noted that there was decreased light-adapted b-wave amplitude on fERG. Moreover, cocaine craving was observed to correlate with a depression in the b-wave amplitude $[12,14,17,18]$. Variable levels of correlation were noted across studies, with calculated r-values ranging from 0.35 to 0.9 .

Studies reporting on cannabis use presented varied parameters. Two articles [5,23] reported an increase in implicit time, with Schwitzer et al. [5] reporting a sensitivity of 78.6\% (95\% CI 60.589.8 ) and a specificity of $75.0 \%$ (95\% CI 55.1-88.1) for an N95 implicit time longer than $93.15 \mathrm{~ms}$ for PERG.

For alcohol consumption, one study [6] found a reduced darkadapted b-wave amplitude on fERG.

\subsection{Alzheimer's disease}

Four studies evaluating differences in ERG waveforms in Alzheimer's disease met our inclusion criteria [25-28], all of which used PERG. No studies were able to determine a statistically significant difference in PERG in patients with Alzheimer's disease, however, a trend was noted towards a reduced b-wave amplitude. Details of studies investigating Alzheimer's disease are shown in Table 2.

\subsection{Autism spectrum disorder}

Similar to Alzheimer's disease, four articles studying ERG in the context of autism spectrum disorder were included in our review [29-32]. Three articles [29,30,32] used a fERG while one used a PERG [31]. Articles using a fERG found a decreased b-wave amplitude (rod function) in patients with autism spectrum disorder, however, quantitative analyses were not provided. Realmuto et al. [30] showed a similar reduction in a families of patients with four siblings and two fathers affected, congruent with the neurodevelopmental origin of autism. Results from studies in this group are shown in Table 3.

\subsection{Depressive disorders}

12 articles evaluating ERG in depressive disorders were eligible [33-44]. Seven of these studied seasonal affective disorder (SAD) and the remaining five major depressive disorder (MDD). There was little consistency in protocol across studies, making crosscomparisons difficult. For MDD, a trend was noted towards decreased contrast gain on PERG, especially for moderate-tosevere depression, although Fam et al. [42], were unable to replicate these findings. Notably, studies also evaluated the impact of interventions on MDD and found that ERG abnormalities resolved with treatment. Similarly, studies evaluating SAD showed variable results on ERG, although there was normalization of irregularities with light therapy or during the summer months [36]. Details of the studies investigating depressive disorders are shown in Table 4.

\subsection{Schizophrenia}

In total, 11 articles investigating ERG in the context of schizophrenia were eligible [45-55]. All but one of the articles used the fERG [45-53,55], with the remaining article implementing PERG [54]. Hébert et al. [50] noted an increase in implicit time in patients with schizophrenia. Across articles, there was also a trend of reduced a- and b-wave amplitudes in schizophrenia. Interestingly, Gerbaldo et al. [46] failed to find this reduction, although their study population differed from that of other articles as it included individuals with a history of sungazing. The most powered study in this group, by Hébert et al. [48], showed reductions in both a- and b-wave amplitudes. Notably, Demmin et al. [55] also provided data on photopic negative response, noting that the schizophrenia group demonstrated attenuated negativity in comparison to healthy controls. Full details of articles investigating ERG in schizophrenia are available in Table 5.

\subsection{Panic disorder}

Two articles evaluating the diagnostic ability of fERG in participants with panic disorder were included in our review $[56,57]$. Both articles found decreased b-wave amplitudes in patients, and reduced differences between right and left eyes in the b-wave amplitudes. The details of these two studies are discussed in Table 6.

\subsection{Eating disorders}

Only two studies evaluating ERG in the context of eating disorders met our inclusion criteria [58,59]. Nasser et al. [58] 


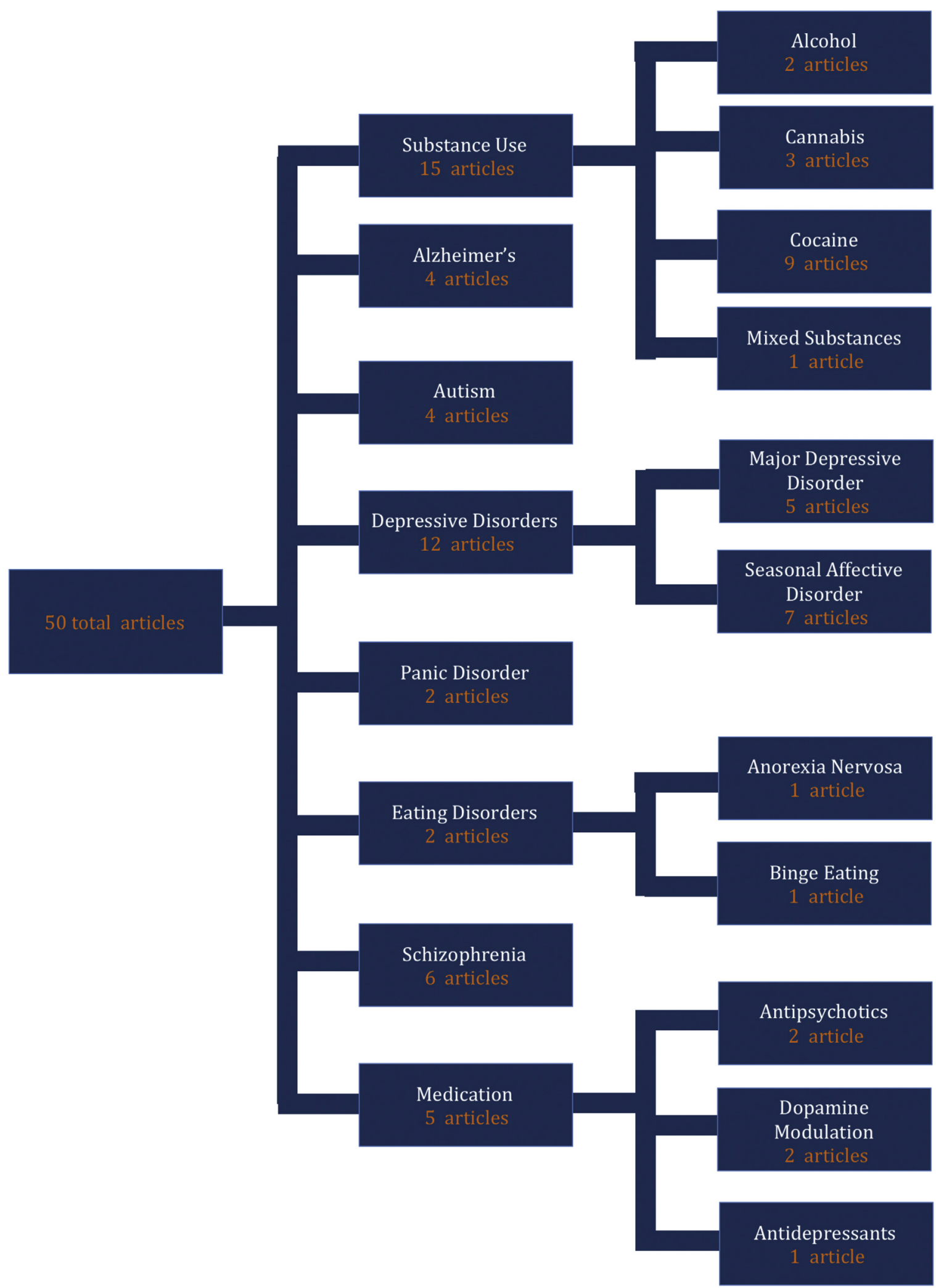

Fig. 3. Number of articles by specific psychiatric illness and topic.

discussed oral food stimulation in the setting of binge eating, while the study by Moschos et al. [59] discussed anorexia nervosa. An increase in b-wave amplitude in response to brownie consumption and a correlation between the Gormally Binge Eating Scale and increased $b$-wave amplitude $(r=0.68)$ was noted on $f E R G$ for binge eating. Moreover, the authors noted that there was a similar increase in b-wave amplitude following administration of methylphenidate [58]. In anorexia nervosa, a decreased P1 retinal response density amplitude was found for ring 1 using mfERG [59]. Complete details of studies in this group are available in Table 7. 


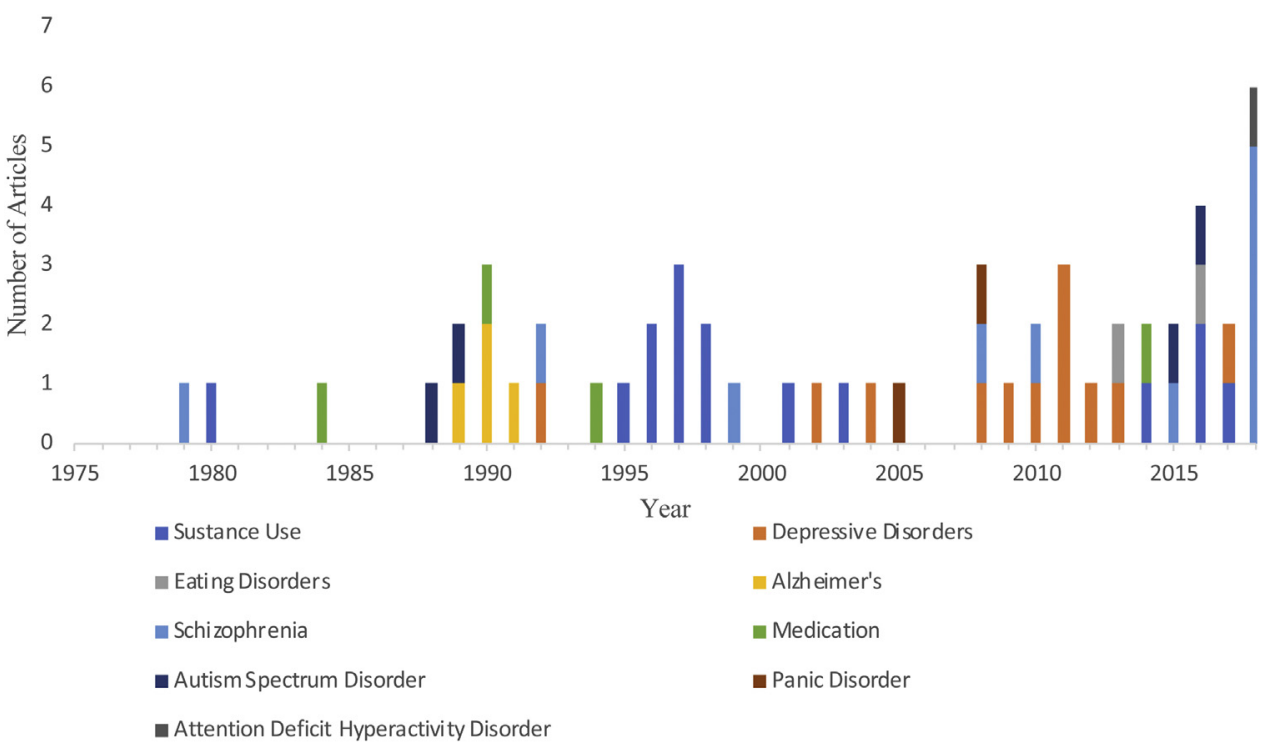

Fig. 4. Timeline of the literature on applications of ERG in psychiatry stratified by specific illness and topic.

Table 1

Articles on substance use.

\begin{tabular}{|c|c|c|c|c|c|c|}
\hline Study & Substance Studied & $\begin{array}{l}\text { Sample } \\
\text { Size }\end{array}$ & $\begin{array}{l}\text { Study } \\
\text { Design }\end{array}$ & ERG Modality & Findings & Photoreceptor \\
\hline Ladien 1980 & Alcohol & - & Letter & fERG & Reduced dark-adapted b-wave amplitude & Not stated \\
\hline Pérez 1995 & $\begin{array}{l}\text { Heroin, Cannabis, } \\
\text { Cocaine }\end{array}$ & 15 & $\begin{array}{l}\text { Cross- } \\
\text { sectional }\end{array}$ & fERG & $\begin{array}{l}\text { Decreased amplitudes and decreased implicit times in more } \\
\text { than } 50 \% \text { of participants compared to controls }\end{array}$ & Not stated \\
\hline Roy 1996 & $\begin{array}{l}\text { Cocaine, recently } \\
\text { withdrawn }\end{array}$ & 14 & $\begin{array}{l}\text { Cross- } \\
\text { sectional }\end{array}$ & fERG, blue filter & $\begin{array}{l}\text { Reduced light-adapted b-wave amplitude in response to blue } \\
\text { light; negative correlation between b-wave amplitude and } \\
\text { cocaine craving ( } r=-0.54)\end{array}$ & Cones \\
\hline $\begin{array}{l}\text { Smelson } \\
1996\end{array}$ & $\begin{array}{l}\text { Cocaine, recently } \\
\text { withdrawn }\end{array}$ & 6 & $\begin{array}{l}\text { Cross- } \\
\text { sectional }\end{array}$ & fERG, blue filter & $\begin{array}{l}\text { Reduced light-adapted b-wave amplitude in response to blue } \\
\text { light; correlation between reduction and cocaine craving } \\
(\mathrm{r}=0.90)\end{array}$ & Cones \\
\hline Roy 1997 & $\begin{array}{l}\text { Cocaine, recently } \\
\text { withdrawn }\end{array}$ & 20 & $\begin{array}{l}\text { Cross- } \\
\text { sectional }\end{array}$ & $\begin{array}{l}\text { fERG, multiple color } \\
\text { filters }\end{array}$ & $\begin{array}{l}\text { Reduced light-adapted b-wave amplitudes, many cocaine } \\
\text { withdrawn participants had amplitudes below } 0.5 \mathrm{mV}\end{array}$ & Cones \\
\hline Roy 1997 & $\begin{array}{l}\text { Cocaine, recently } \\
\text { withdrawn }\end{array}$ & 30 & $\begin{array}{l}\text { Cross- } \\
\text { sectional }\end{array}$ & fERG, blue filter & $\begin{array}{l}\text { Negative correlation between blue cone } b \text {-wave amplitude and } \\
\text { scores on cocaine craving questionnaire }(r=-0.35)\end{array}$ & Cones \\
\hline Roy 1997 & Cocaine, withdrawn & 8 & Longitudinal & fERG, blue filter & $\begin{array}{l}\text { No difference in light-adapted b-wave amplitude over duration } \\
\text { of study }\end{array}$ & Cones \\
\hline Roy 1998 & $\begin{array}{l}\text { Cocaine, recently } \\
\text { withdrawn }\end{array}$ & 8 & Longitudinal & fERG, blue filter & $\begin{array}{l}\text { No increase in light-adapted b-wave amplitude with } \\
\text { risperidone }\end{array}$ & Cones \\
\hline $\begin{array}{l}\text { Smelson } \\
1998\end{array}$ & $\begin{array}{l}\text { Cocaine, recently } \\
\text { withdrawn }\end{array}$ & 14 & $\begin{array}{l}\text { Cross- } \\
\text { sectional }\end{array}$ & fERG, blue filter & $\begin{array}{l}\text { Reduced light-adapted b-wave amplitude correlated with } \\
\text { higher cocaine craving }\end{array}$ & Cones \\
\hline $\begin{array}{l}\text { Smelson } \\
2001\end{array}$ & $\begin{array}{l}\text { Cocaine, recently } \\
\text { withdrawn }\end{array}$ & 21 & $\begin{array}{l}\text { Cross- } \\
\text { sectional }\end{array}$ & $\begin{array}{l}\text { fERG, multiple color } \\
\text { filters }\end{array}$ & $\begin{array}{l}\text { Participants with lower light-adapted b-wave amplitudes had } \\
\text { higher craving scores }\end{array}$ & Cones \\
\hline Roy 2003 & $\begin{array}{l}\text { Cocaine, recently } \\
\text { withdrawn }\end{array}$ & 17 & $\begin{array}{l}\text { Cross- } \\
\text { sectional }\end{array}$ & $\begin{array}{l}\text { fERG, blue and red } \\
\text { filters }\end{array}$ & $\begin{array}{l}\text { Lower light-adapted b-wave amplitude correlated with lower } \\
\text { CSF homovanillic acid (dopamine metabolite) }(r=0.57)\end{array}$ & Cones \\
\hline $\begin{array}{l}\text { Schwitzer } \\
2014\end{array}$ & Cannabis & - & $\begin{array}{l}\text { Cross- } \\
\text { sectional }\end{array}$ & fERG & $\begin{array}{l}\text { Increased implicit time for a- and b-waves in light- and dark- } \\
\text { adapted conditions }\end{array}$ & Not stated \\
\hline Kim 2016 & Alcohol & 15 & Prospective & mfERG & Shortening of P1 implicit time of ring 1 & Cones \\
\hline $\begin{array}{l}\text { Schwitzer } \\
2016\end{array}$ & Cannabis & 1 & Case study & fERG & $\begin{array}{l}\text { Decreased dark-adapted a-wave amplitude } 30 \text { minutes after } \\
\text { smoking }\end{array}$ & Not stated \\
\hline $\begin{array}{l}\text { Schwitzer } \\
2017\end{array}$ & Cannabis & 28 & $\begin{array}{l}\text { Cross- } \\
\text { sectional }\end{array}$ & PERG & $\begin{array}{l}\text { Increase in N95 implicit time; suggest that an N95 implicit time } \\
\text { longer than } 91.13 \text { ms demonstrates regular cannabis use: } \\
\text { sensitivity } 78.6 \% \text { ( } 95 \% \text { CI 60.5-89.8), specificity } 75.0 \% \text { (95\% CI } \\
55.1-88.0 \text { ) }\end{array}$ & Not stated \\
\hline
\end{tabular}

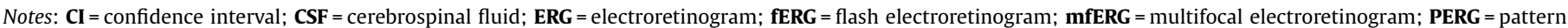
electroretinogram.

\subsection{Attention deficit hyperactivity disorder}

One article discussing attention deficit hyperactivity disorder (ADHD) was eligible, and it utilized the PERG [60]. Treatment with methylphenidate was also discussed. The authors found that there was increased background noise on PERG in patients and that this normalized with treatment. Table 8 includes details of the article investigating ERG in ADHD.

\subsection{Medication}

Five articles assessed the effects of medication on healthy participants by ERG [61-65]. Two articles found that perphenazine decreased b-wave amplitudes, while bromocriptine increased them [62,63]. Several dopamine antagonists such as haloperidol, chlorpromazine, fluphenazine, and metoclopramide, reduced b-wave amplitudes, although these decreases were not 
Table 2

Articles on Alzheimer's disease.

\begin{tabular}{|c|c|c|c|c|c|}
\hline Study & $\begin{array}{l}\text { Sample } \\
\text { Size }\end{array}$ & Study Design & $\begin{array}{l}\text { ERG } \\
\text { Modality }\end{array}$ & Findings & Photoreceptor \\
\hline Katz 1989 & 6 & Cross-sectional & PERG & $\begin{array}{l}\text { B-wave amplitude was reduced by half in participants with } \\
\text { Alzheimer's disease, no difference in latencies }\end{array}$ & Not stated \\
\hline Pollock 1990 & - & Letter, response to Katz et al., 1989 & PERG & $\begin{array}{l}\text { Katz } 1989 \text { had a small sample size and cannot conclude } \\
\text { statistical significance }\end{array}$ & Not stated \\
\hline Katz 1990 & - & $\begin{array}{l}\text { Letter, response to Pollock \& } \\
\text { Schneider } 1990\end{array}$ & PERG & $\begin{array}{l}\text { PERG was reduced in participants with Alzheimer's but not } \\
\text { statistically significant }\end{array}$ & Not stated \\
\hline Strenn 1991 & 8 & Cross-sectional & PERG & No anomalies noted & Not stated \\
\hline
\end{tabular}

Notes: $\mathbf{P E R G}=$ pattern electroretinogram.

Table 3

Articles on autism spectrum disorder.

\begin{tabular}{|c|c|c|c|c|c|}
\hline Study & Sample Size & Study Design & ERG Modality & Findings & Photoreceptor \\
\hline Ritvo 1988 & 27 & Cross-sectional & $\begin{array}{l}\text { fERG, blue } \\
\text { and red filters }\end{array}$ & $13 / 27$ participants ( $48 \%$ ) had below normal b-wave amplitudes & Not stated \\
\hline Realmuto 1989 & 10 & Cross-sectional & fERG & $6 / 10$ participants (60\%) had decreased dark-adapted b-wave amplitudes & Cones and rods \\
\hline van Elst 2015 & 33 & Cross-sectional & PERG & No differences between disease and healthy groups & $\begin{array}{l}\text { No photoreceptor - } \\
\text { ganglion cell involved }\end{array}$ \\
\hline Constable 2016 & 11 & Cross-sectional & fERG & $\begin{array}{l}\text { Decreased light- and dark-adapted b-wave amplitudes, however, } \\
\text { dark-adapted b-wave amplitudes not as low Ritvo } 1988\end{array}$ & Cones and rods \\
\hline
\end{tabular}

Notes: $\mathbf{f E R G ~ = ~ f l a s h ~ e l e c t r o r e t i n o g r a m ; ~} \mathbf{P E R G ~ = ~ p a t t e r n ~ e l e c t r o r e t i n o g r a m . ~}$

Table 4

Articles on depressive disorders.

\begin{tabular}{|c|c|c|c|c|c|c|}
\hline Study & $\begin{array}{l}\text { Disorder } \\
\text { Studied }\end{array}$ & $\begin{array}{l}\text { Sample } \\
\text { Size }\end{array}$ & Study Design & $\begin{array}{l}\text { ERG } \\
\text { Modality }\end{array}$ & Findings & Photoreceptor \\
\hline Lam 1992 & SAD & 24 & Cross-sectional & fERG & $\begin{array}{l}\text { Males had higher b-waves than control, while females had } \\
\text { lower b-waves than controls; implicit time longer in left eyes of } \\
\text { males }\end{array}$ & $\begin{array}{l}\text { Cones and } \\
\text { rods }\end{array}$ \\
\hline Hébert 2002 & $\begin{array}{l}\text { SAD, sub- } \\
\text { syndromal }\end{array}$ & 12 & Longitudinal & fERG & $\begin{array}{l}\text { Changes in Global Seasonality Score were reflected in retinal } \\
\text { sensitivity in SAD participants, SSAD participants showed } \\
\text { differences between winter and summer response curves }\end{array}$ & Not stated \\
\hline Hébert 2004 & SAD & 27 & Cross-sectional & fERG & $\begin{array}{l}40 \% \text { of SAD participants had dark-adapted light sensitivity } \\
\text { (logK) below } 1 \text { SD that of the control group }\end{array}$ & Not stated \\
\hline $\begin{array}{l}\text { Danilenko } \\
2008\end{array}$ & SAD & 22 & Cross-sectional & fERG & $\begin{array}{l}\text { Diet had no effect, dark-adapted amplitude diminished after } \\
\text { eating for all groups }\end{array}$ & $\begin{array}{l}\text { Cones and } \\
\text { rods }\end{array}$ \\
\hline Lavoie 2009 & SAD & 22 & Longitudinal & fERG & $\begin{array}{l}\text { Light-adapted ERG had lower amplitude and light sensitivity } \\
\text { before light therapy in SAD participants which normalized after } \\
\text { treatment; b-wave implicit time } 7 \% \text { longer in winter months } \\
\text { than summer months in SAD participants; dark-adapted } \\
\text { sensitivity increased with treatment }\end{array}$ & $\begin{array}{l}\text { Cones and } \\
\text { rods }\end{array}$ \\
\hline Bubl 2010 & MDD & 40 & Cross-sectional & PERG & $\begin{array}{l}\text { Below-normal PERG contrast gain in MDD participants } \\
\text { compared to control; below-normal contrast gain predicts low } \\
\text { score on HAM-D with specificity of } 92.5 \% \text { and sensitivity of } \\
77.5 \%\end{array}$ & Not stated \\
\hline Fornaro 2011 & MDD & 20 & Longitudinal & fERG & $\begin{array}{l}\text { Participants who responded to treatment had higher baseline } \\
\text { ERG which normalized within the } 12 \text { weeks }\end{array}$ & $\begin{array}{l}\text { Cones and } \\
\text { rods }\end{array}$ \\
\hline Gagne 2011 & SAD & 12 & Longitudinal & fERG & $\begin{array}{l}\text { SAD participants had lower light-adapted a-wave amplitudes, } \\
\text { amplitudes for this group was higher in summer than in winter, } \\
\text { SAD patients had lower b-wave dark-adapted VMax amplitudes } \\
\text { for } 5 \text { and } 10000 \text { lux exposures }\end{array}$ & $\begin{array}{l}\text { Cones and } \\
\text { rods }\end{array}$ \\
\hline $\begin{array}{l}\text { Vandewalle } \\
2011\end{array}$ & SAD & 14 & Cross-sectional & fERG & No difference in light sensitivity (logK) & $\begin{array}{l}\text { Cones and } \\
\text { rods }\end{array}$ \\
\hline Bubl 2012 & MDD & 14 & Longitudinal & PERG & $\begin{array}{l}\text { At baseline, MDD participants had lower contrast gain than } \\
\text { controls, this normalized with treatment; if HAM-D is greater } \\
\text { than or equal to } 7 \text {, and BDI score is between } 10-11 \text { (moderate- } \\
\text { severe depression), then sensitivity and specificity is } 100 \%\end{array}$ & Not stated \\
\hline Fam 2013 & MDD & 20 & Cross-sectional & $\begin{array}{l}\text { fERG and } \\
\text { PERG }\end{array}$ & No significant differences between groups & Not stated \\
\hline Hébert 2017 & MDD & 100 & Cross-sectional & fERG & $\begin{array}{l}\text { Light-adapted b-wave implicit time was prolonged in } \\
\text { participants with MDD, b-wave VMax was reached at a lower } \\
\text { luminance in non-medicated participants than in medicated } \\
\text { participants }\end{array}$ & $\begin{array}{l}\text { Cones and } \\
\text { rods }\end{array}$ \\
\hline
\end{tabular}

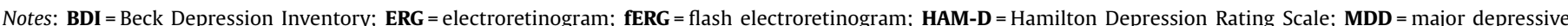
disorder; PERG = pattern electroretinogram; SAD = seasonal affective disorder; SSAD = sub-syndromal seasonal affective disorder. 
Table 5

Articles on schizophrenia.

\begin{tabular}{|c|c|c|c|c|c|}
\hline Study & Sample Size & Study Design & ERG Modality & Findings & Photoreceptor \\
\hline Gagrat 1979 & 3 & Case series & fERG & No differences before and after treatment & Not stated \\
\hline Gerbaldo 1992 & 9 & Cross-sectional & fERG & $\begin{array}{l}\text { No difference between participants with schizophrenia and } \\
\text { controls; participants with history of sun gazing had decreased } \\
\text { light-adapted retinal sensitivity }\end{array}$ & Not stated \\
\hline Warner 1999 & 9 & Cross-sectional & fERG & $\begin{array}{l}\text { Reduced dark- and light-adapted a- and b-waves (both cone and } \\
\text { rod) }\end{array}$ & Cones and rods \\
\hline Balogh 2008 & 26 & Longitudinal & fERG & $\begin{array}{l}\text { Reduced a-wave amplitude; as positive PANSS scores increased, } \\
\text { a-wave amplitude decreased for participants with } \\
\text { schizophrenia ( } r=-0.51 \text { ) for cones only during the acute phase } \\
\text { of the disease }\end{array}$ & Cones \\
\hline Hébert 2010 & 29 & Cross-sectional & fERG & Reduced dark-adapted b-wave VMax amplitude & Cones and rods \\
\hline Hébert 2015 & 105 & Cross-sectional & fERG & Reduced a-and b-wave amplitudes at the cone & Cones and rods \\
\hline Gagné 2018 & 33 & Longitudinal & fERG & $\begin{array}{l}\text { Reduced dark-adapted b-wave amplitude; large variation in } \\
\text { ERG over time }\end{array}$ & Cones and rods \\
\hline Maziade 2018 & 84 & Cross-sectional & fERG & $\begin{array}{l}\text { Lengthened light-adapted b-wave latency, reduced dark- } \\
\text { adapted b-wave amplitude and latency }\end{array}$ & Cones and rods \\
\hline Silverstein 2018 & 25 & Cross-sectional & fERG & Weaker photoreceptor response & $\begin{array}{l}\text { Photoreceptor type not stated - } \\
\text { bipolar and ganglion cells involved }\end{array}$ \\
\hline Laprevote 2018 & 15 & Cross-sectional & PERG & $\begin{array}{l}\text { Increased N95 implicit time for patients with visual } \\
\text { hallucinations }\end{array}$ & Not stated \\
\hline Demmin 2018 & 25 & Cross-sectional & fERG & $\begin{array}{l}\text { Reduced dark- and light-adapted a- and b-wave amplitude, } \\
\text { reduced amplitudes for a flicker test; attenuated negativity of } \\
\text { photopic negative response }\end{array}$ & Cones and rods \\
\hline
\end{tabular}

Notes: $\mathbf{E R G}=$ electroretinogram; fERG = flash electroretinogram; PANSS = Positive and Negative Syndrome Scale; PERG= pattern electroretinogram.

Table 6

Articles on panic disorder.

\begin{tabular}{|c|c|c|c|c|c|}
\hline Study & Sample Size & Study Design & ERG Modality & Findings & Photoreceptor \\
\hline Pieraccini 2005 & 29 & Cross-sectional & fERG & $\begin{array}{l}\text { Decreased b-wave amplitude; differences in b-wave amplitudes between } \\
\text { eyes reduced }\end{array}$ & Not stated \\
\hline Bossini 2008 & 22 & Cross-sectional & fERG & $\begin{array}{l}\text { Decreased b-wave amplitude; difference between b-wave amplitudes in right } \\
\text { and left eyes decreased }\end{array}$ & Not stated \\
\hline
\end{tabular}

Notes. fERG= flash electroretinogram.

Table 7

Articles on eating disorders.

\begin{tabular}{|c|c|c|c|c|c|c|}
\hline Study & Disorder Studied & Sample Size & Study Design & ERG Modality & Findings & Photoreceptor \\
\hline Nasser 2013 & $\begin{array}{l}\text { Binge eating, } \\
\text { oral food } \\
\text { stimulation }\end{array}$ & 9 & Cross-sectional & fERG & $\begin{array}{l}\text { Increase in b-wave amplitude for brownie consumption and } \\
\text { correlation between Gormally Binge Eating Scale and increase } \\
\text { in b-wave amplitude }(r=0.68) \text {; correlation between Stunkard } \\
\text { and Messick Three Factor Eating Questionnaire and increase in } \\
\text { b-wave amplitude }(r=0.67)\end{array}$ & Cones \\
\hline Moschos 2016 & Anorexia nervosa & 13 & Cross-sectional & mfERG & Lower P1 retinal response density amplitude for ring 1 & Not stated \\
\hline
\end{tabular}

Notes. $\mathbf{f E R G}=$ flash electroretinogram; $\mathbf{m f E R G =}$ multifocal electroretinogram.

Table 8

Articles on ADHD.

\begin{tabular}{|c|c|c|c|c|c|}
\hline Study & Sample Size & Study Design & ERG Modality & Findings & Photoreceptor \\
\hline Bubl 2018 & 20 & Longitudinal & PERG & $\begin{array}{l}\text { Elevated background noise (127\%) before treatment, which normalized } \\
\text { after methylphenidate administration; background noise correlated } \\
\text { with severity of ADHD symptoms }\end{array}$ & Cones and rods \\
\hline
\end{tabular}

Notes. $\mathbf{A D H D}=$ attention deficit hyperactivity disorder; $\mathbf{P E R G}=$ pattern electroretinogram.

quantified $[63,64]$. Details of studies in this group are discussed in Table 9.

\section{Discussion}

The present review aimed to evaluate the utility of ERG as a diagnostic tool within a psychiatric setting in a systematic manner. Because of the lack of consistency amongst protocols, small sample sizes, and lack of replicable findings, there remains much work that needs to be done before ERG may be used reliably in psychiatry. The major findings of our review are summarized in Table 10.

In performing our review, we found it exceedingly difficult to make comparisons across studies and draw conclusions for specific disease groups. There was little consistency between protocols in included articles, and studies within disease groups often examined distinct forms of therapy and were conducted over drastically different timelines, leading to variable results. Moreover, many studies did not quantify their results, making it 
Table 9

Articles on medication use.

\begin{tabular}{|c|c|c|c|c|c|c|}
\hline Study & Medication (Class) & $\begin{array}{l}\text { Sample } \\
\text { Size }\end{array}$ & Study Design & $\begin{array}{l}\text { ERG } \\
\text { Modality }\end{array}$ & Findings & Photoreceptor \\
\hline Filip 1978 & Thioridazine (typical antipsychotic) & 10 & Cross-sectional & fERG & $\begin{array}{l}\text { Drug intake prolonged a-wave latency and } \\
\text { b-wave evolution time, while decreasing } \\
\text { b-wave amplitude }\end{array}$ & Not stated \\
\hline Fornaro 1984 & $\begin{array}{l}\text { Perphenazine (typical antipsychotic), } \\
\text { Bromocriptine (dopamine agonist) }\end{array}$ & 18 & Cross-sectional & fERG & $\begin{array}{l}\text { Perphenazine decreased b-wave amplitude; } \\
\text { bromocriptine increased b-wave amplitude }\end{array}$ & Not stated \\
\hline Perossini 1990 & $\begin{array}{l}\text { Perphenazine (typical antipsychotic), } \\
\text { Haloperidol (typical antipsychotic), Levodopa/ } \\
\text { Carbidopa (dopaminergic), Nomifensine } \\
\text { (NDRI), Bromocriptidine (dopamine agonist), } \\
\text { Imipramine (TCA), Diazepam } \\
\text { (benzodiazepine) }\end{array}$ & 60 & Cross-sectional & fERG & $\begin{array}{l}\text { Perphenazine decreased b-wave amplitude; } \\
\text { bromocriptine increased b-wave amplitude; } \\
\text { Nomifensine increased b-wave amplitude, } \\
\text { Levodopa/Carbidopa increased b-wave } \\
\text { amplitude; Haloperidol decreased b-wave } \\
\text { amplitude; Imipramine and Diazepam had } \\
\text { no effect }\end{array}$ & Not stated \\
\hline $\begin{array}{l}\text { Holopigian } \\
1994\end{array}$ & $\begin{array}{l}\text { Chlorpromazine (typical antipsychotic), } \\
\text { Fluphenazine (typical antipsychotic), } \\
\text { Metoclopramide (dopamine antagonist) }\end{array}$ & 22 & Cross-sectional & fERG & $\begin{array}{l}\text { Reduced b-wave amplitudes and amplitudes } \\
\text { for first oscillatory potential }\end{array}$ & $\begin{array}{l}\text { Cones and } \\
\text { rods }\end{array}$ \\
\hline Fornaro 2014 & Agomelatine (atypical antidepressant) & 23 & Cross-sectional & fERG & $\begin{array}{l}\text { Slight increase in b-wave amplitude and } \\
\text { latency in both eyes }\end{array}$ & Cones \\
\hline
\end{tabular}

Notes: $\mathbf{f E R G ~ = ~ f l a s h ~ e l e c t r o r e t i n o g r a m ; ~ N D R I ~ = ~ n o r e p i n e p h r i n e - d o p a m i n e ~ r e u p t a k e ~ i n h i b i t o r ; ~} \mathbf{T C A}$ = tricyclic antidepressant.

Table 10

Summary of ERG findings across psychiatric illnesses and groups.

\begin{tabular}{|c|c|}
\hline Group & Finding(s) \\
\hline Cocaine withdrawal & $\begin{array}{l}\text { Reduced light-adapted b-wave in response } \\
\text { to blue light, reduction correlated with } \\
\text { cocaine craving }\end{array}$ \\
\hline Cannabis use & Increased implicit time \\
\hline Alcohol use & - \\
\hline Alzheimer's disease & - \\
\hline Autism spectrum disorder & Reduced b-wave amplitude \\
\hline MDD & $\begin{array}{l}\text { Reduced contrast gain in PERG, PERG } \\
\text { normalization with treatment }\end{array}$ \\
\hline SAD & $\begin{array}{l}\text { Various abnormalities that normalize with } \\
\text { remission }\end{array}$ \\
\hline Schizophrenia & Reduced a- and b-wave amplitudes \\
\hline Panic disorder & $\begin{array}{l}\text { Reduced b-wave amplitudes, Reduced } \\
\text { differences between right and left eyes in } \\
\text { b-wave amplitudes }\end{array}$ \\
\hline Eating disorders & - \\
\hline ADHD & - \\
\hline Perphenazine use & Reduced b-wave amplitudes \\
\hline Bromocriptine use & Increased b-wave amplitudes \\
\hline Dopamine antagonists & Reduced b-wave amplitudes \\
\hline
\end{tabular}

Notes: $\mathbf{A D H D}=$ attention deficit hyperactivity disorder; $\mathbf{E R G}=$ electroretinogram; $\mathbf{M D D}=$ major depressive disorder; $\mathbf{P E R G}=$ pattern electroretinogram; $\mathbf{S A D}=$ seasonal affective disorder.

challenging to draw comparisons and eliminating any possibility of aggregating results meaningfully through a meta-analysis. While qualitative observations of waveform reductions and increases are insightful at an exploratory level, they do not provide an objective assessment of diagnostic utility.

In order for ERG to become a diagnostic modality in psychiatry, there is a need for quantifiable data that allows for determination of important measures such as sensitivity, specificity, and positive and negative predictive values in comparison to gold standard diagnostic methods. To facilitate this, we suggest that future studies implement the International Society for Clinical Electrophysiology of Vision (ISCEV) guidelines for the measurement of ERG waveforms and should quantify anomalies and calculate the magnitude of correlations with disease states. Prior studies were largely underpowered due to a small sample size. This may have been a result of requiring trained technicians and nurses to carry out pharmacological pupil dilation prior to ERG. Newer ERG technology permits gathering of data without the need for pupil dilation. Thus, authors should ensure that their studies have sufficient sample sizes and are adequately powered to assess outcomes of interest. To enrich results and minimize bias, future studies should be multi-center. At present, most of the research on a particular condition is conducted by the same authors. For instance, of 12 articles on depressive disorders, seven were from the same research group, and out of 15 articles on substance use, nine were from the same group of authors. Having multiple centers participate in studies would serve to both internally and externally validate study protocol and improve the reliability of findings.

Future studies should also consider expanding the use of ERG to yet unexplored psychiatric conditions. For example, our search did not identify any eligible studies examining bipolar disorder, indicating a gap in the present evidence.

An inherent difficulty of using ERG as a diagnostic tool in psychiatry is the limited number of detectable anomalies, restricting diagnostic specificity. For example, a reduction in bwave amplitude on fERG may be indicative of cocaine withdrawal, autism spectrum disorder, panic disorder, perphenazine use, or intake of dopamine blockers. From a neurobiological perspective, this is perhaps due to the high degree of interconnectedness between many neurotransmitter systems [66]. Emerging research in animal suggests that ERG responses can also be affected by dopamine, accounting for observed anomalies in relevant psychiatric illnesses and medication use [2]. To be used reliably as a diagnostic tool, ERG will have to demonstrate more particular anomalies for each condition. If this proves to be unfeasible, however, then the diagnostic utility of ERG may be limited and its use in psychiatry may be better suited to measuring the effect of treatments or predicting relapse in patients.

\section{Limitations}

Our review is not without its limitations. We do not present quantitative analyses or a meta-analysis summarizing the results of ERG anomalies in specific psychiatric conditions as included studies did not provide such data.

Moreover, our review is at risk for publication bias as many of the studies included were conducted by a limited number of research groups and were largely single-center in design. Our review is also limited by its broad research question. There is a paucity of literature when examining the applicability of ERG to specific psychiatric illnesses, and thus we chose to look at a myriad of pathologies in order to provide the most comprehensive summary of the current evidence. 
As the pace of research in this field accelerates, we anticipate future reviews will have well-refined and focused research questions and will allow for quantitative analyses.

\section{Conclusions}

This review systematically examined the literature on the use of ERG as a diagnostic tool in psychiatry. Although it was difficult to draw quantitative conclusions, consistent trends in ERG waveform anomalies in specific psychiatric conditions were observed across included studies. ERG is a non-invasive, quickly administered, and well-characterized test that has the potential to become an objective tool for the diagnosis of psychiatric illness. Further investigation through adequately powered multi-center studies, in concordance with the rapid pace of technological advancement, will allow for thorough evaluation of ERG in comparison to existing gold standard modalities and permit its successful integration into the diagnostic repertoire of modern psychiatry.

\section{Funding}

This research did not receive any specific grant from funding agencies in the public, commercial, or not-for-profit sectors.

\section{References}

[1] Lavoie J, Maziade M, Hébert M. The brain through the retina: the flash electroretinogram as a tool to investigate psychiatric disorders. Prog Neuropsychopharmacol Biol Psych 2014;48:129-34.

[2] Lavoie J, Illiano P, Sotnikova TD, Gainetdinov RR, Beaulieu JM, Hébert M. The electroretinogram as a biomarker of central dopamine and serotonin: potential relevance to psychiatric disorders. Biol Psychiatry 2014;75(6):47986.

[3] Brown KT. The electroretinogram: its components and their origins. Vision Res 1968;8(6):633-77.

[4] McCulloch DL, Marmor MF, Brigell MG. ISCEV Standard for full-field clinical electroretinography (2015 update). Doc Ophthalmol 2015;130(1):1-12.

[5] Schwitzer T, Schwan R, Angioi-Duprez K, Giersch A, Laprevote V. The endocannabinoid system in the retina: from physiology to practical and therapeutic applications. Neural Plast 2016 2016;1-10.

[6] Ladien K, Levett J, Clarke M. Effects of alcohol on electroretinogram light/dark adaptation. Drug Alcohol Depend 1980;6(1):93-5.

[7] Hildebrand GD, Fielder AR. Anatomy and physiology of the retina. In: Reynolds J, Olitsky SE, editors. Pediatric retina. New York: Springer-Verlag Berlin Heidelberg; 2011. p. 39-65.

[8] Bach M, Brigell MG, Hawlina M, et al. ISCEV standard for clinical pattern electroretinography (PERG) 2012 update. Doc Ophthalmol 2013;126(1):1-7.

[9] Hood DC, Bach M, Brigell M, et al. International society for clinical electrophysiology of vision. ISCEV standard for clinical multifocal electroretinography (mfERG) (2011 ed.). Doc Ophthalmol 2012;124(1):1-3.

[10] McInnes MDF, Moher D, Thombs BD, et al. Preferred reporting items for a systematic review and meta-analysis of diagnostic test accuracy studies: the PRISMA-DTA statement. JAMA 2018;319(4):388-96.

[11] Pérez JG, Mato MP, García AS, Rey AD. Intraocular motility, electrophysiologica tests and visual fields in drug addicts. Ophthalmic Physiol Opt 1995;15 (5):493-8.

[12] Roy M, Smelson DA, Roy A. Abnormal electroretinogram in cocaine-dependent patients. Relationship to craving. Br J Psychiatry 1996;168(4):507-11.

[13] Roy M, Roy A, Williams J, Weinberger L, Smelson D. Reduced blue cone electroretinogram in cocaine-withdrawn patients. Arch Gen Psychiatry 1997:54(2):153-6.

[14] Roy M, Roy A, Smelson D, Brown S, Weinberger L. Reduced blue cone electroretinogram in withdrawn cocaine dependent patients: a replication. Biol Psychiatry 1997;42(7):631-3.

[15] Roy M, Smelson D, Roy A. Longitudinal study of blue cone retinal function in cocaine-withdrawn patients. Biol Psychiatry 1997;41(2):252-3.

[16] Roy A, Roy M, Smelson DA. Risperidone, ERG, and cocaine craving. Am J Addict 1998;7(1):90.

[17] Roy A, Roy M, Berman J, Gonzalez B. Blue cone electroretinogram amplitudes are related to dopamine function in cocaine-dependent patients. Psychiatry Res 2003;117(2):191-5.

[18] Smelson DA, Roy A, Roy M. The electroretinogram and neuropsychological functioning in cocaine addicts. Can J Psychiatry 1996;41(6):415.

[19] Smelson DA, Roy M, Roy A, Santana S. Electroretinogram in withdrawn cocaine-dependent subjects: relationship to cue-elicited craving. $\mathrm{Br}$ J Psychiatry 1998;172(6):537-9.
[20] Smelson DA, Roy A, Roy M, Tershakovec D, Engelhart C, Losonczy MF Electroretinogram and cue-elicited craving in withdrawn cocaine dependent patients: a replication. Am J Drug Alcohol Abuse 2001;27(2):391-7.

[21] Schwitzer T, Ingster-Moati I, Angioi K, Giersch A, Schwan R, Laprevote V. Impaired retinal processing in regular cannabis users: potential benefit of electroretinogram as a biomark. Eur Psychiatry 2014;29(8):529-30.

[22] Schwitzer T, Robert MP, Giersch A, et al. Transient retinal dysfunctions after acute cannabis use. Eur Addict Res 2016;22(6):287-91.

[23] Schwitzer T, Schwan R, Albuisson E, et al. Association between regular cannabis use and ganglion cell dysfunction. JAMA Ophthalmol 2017;135 (1):54-60.

[24] Kim JT, Yun CM, Kim SW, Oh J, Huh K. The effects of alcohol on visual evoked potential and multifocal electroretinography. J Korean Med Sci 2016;31 (5):783-9.

[25] Katz B, Rimmer S, Iragui V, Katzman R. Abnormal pattern electroretinogram in Alzheimer's disease: evidence for retinal ganglion cell degeneration? Ann Neurol 1989;26(2):221-5.

[26] Katz B, Rimmer S, Klauber MR, Katzman R. The electroretinogram in Alzheimer's disease (Reply). Ann Neurol 1990;28(5):725.

[27] Pollock VE, Schneider LS. The electroretinogram in Alzheimer's disease. Ann Neurol 1990;28(5):724-5.

[28] Strenn K, Dal-Bianco P, Weghaupt H, Koch G, Vass C, Gottlob I. Pattern electroretinogram and luminance electroretinogram in Alzheimer's disease. J Neural Transm 1991;33(suppl):73-80.

[29] Ritvo ER, Creel D, Realmuto G, Crandall AS, Freeman BJ. Electroretinograms in autism: a pilot study of b-wave amplitudes. Am J Psychiatry 1988;145(2):22932.

[30] Realmuto G, Purple R, Knobloch W, Ritvo E. Electroretinograms (ERGs) in four autistic probands and six first-degree relatives. Can J Psychiatry 1989;34 (5):435-9.

[31] van Elst LT, Bach M, Blessing J, Riedel A, Bubl E. Normal visual acuity and electrophysiological contrast gain in adults with high-functioning autism spectrum disorder. Front Hum Neurosci 2015;9:460.

[32] Constable PA, Gaigg SB, Bowler DM, Jägle H, Thompson DA. Full-field electroretinogram in autism spectrum disorder. Doc Ophthalmol 2016;132 (2):83-99.

[33] Lam RW, Beattie CW, Buchanan A, Mador JA. Electroretinography in seasonal affective disorder. Psychiatr Res 1992;43(1):55-63.

[34] Hébert M, Dumont M, Lachapelle P. Electrophysiological evidence suggesting a seasonal modulation of retinal sensitivity in subsyndromal winter depression. J Affect Disord 2002;68(2):191-202.

[35] Danilenko KV, Plisov IL, Hebert M, Kräuchi K, Wirz-Justice A. Influence of timed nutrient diet on depression and light sensitivity in seasonal affective disorder. Chronobiol Int 2008;25(1):51-64.

[36] Lavoie MP, Lam RW, Bouchard G, et al. Evidence of a biological effect of light therapy on the retina of patients with seasonal affective disorder. Biol Psychiatry 2009;66(3):253-8.

[37] Bubl E, Kern E, Ebert D, Bach M, van Elst LT. Seeing gray when feeling blue? Depression can be measured in the eye of the diseased. Biol Psychiatry 2010;68 (2):205-8.

[38] Bubl E, Ebert D, Kern E, van Elst LT, Bach M. Effect of antidepressive therapy on retinal contrast processing in depressive disorder. Br J Psychiatry 2012;201 (2):151-8.

[39] Fornaro M, Bandini F, Ogliastro C, et al. Electroretinographic assessment in major depressed patients receiving duloxetine: Might differences between responders and non-responders indicate a differential biological background? J Affect Disord 2011;135(1):154-9.

[40] Gagné AM, Hébert M. Atypical pattern of rod electroretinogram modulation by recent light history: a possible biomarker of seasonal affective disorder. Psychiatr Res 2011:187(3):370-4.

[41] Vandewalle G, Hébert M, Beaulieu C, et al. Abnormal hypothalamic response to light in seasonal affective disorder. Biol Psychiatry 2011;70(10):954-61.

[42] Fam J, Rush AJ, Haaland B, Barbier S, Luu C. Visual contrast sensitivity in major depressive disorder. J Psychosom Res 2013;75(1):83-6.

[43] Hébert M, Mérette C, Paccalet T, Gagné AM, Maziade M. Electroretinographic anomalies in medicated and drug free patients with major depression: tagging the developmental roots of major psychiatric disorders. Prog Neuropsychopharmacol Biol Psych 2017;75:10-5.

[44] Hébert M, Beattie CW, Tam EM, Yatham LN, Lam RW. Electroretinography in patients with winter seasonal affective disorder. Psychiatr Res 2004;127 (1):27-34.

[45] Gagrat D, Maggiano J, Belmaker RH. Effect of neuroleptic treatment in schizophrenia on the electroretinogram, electrooculogram, and color vision. Psychiatr Res 1979;1(1):109.

[46] Gerbaldo H, Thaker G, Tittel PG, Layne-Gedge J, Moran M, Demisch L. Abnormal electroretinography in schizophrenic patients with a history of sun gazing. Neuropsychobiol 1992;25(2):99-101.

[47] Warner R, Laugharne J, Peet M, Brown L, Rogers N. Retinal function as a marker for cell membrane omega-3 fatty acid depletion in schizophrenia: a pilot study. Biol Psychiatry 1999;45(9):1138-42.

[48] Balogh Z, Benedek G, Kéri S. Retinal dysfunctions in schizophrenia. Prog Neuropsychopharmacol Biol Psych 2008;32(1):297-300.

[49] Hébert M, Gagné AM, Paradis ME, et al. Retinal response to light in young nonaffected offspring at high genetic risk of neuropsychiatric brain disorders. Biol Psychiatry 2010;67(3):270-4. 
[50] Hébert M, Mérette C, Paccalet T, et al. Light evoked potentials measured by electroretinogram may tap into the neurodevelopmental roots of schizophrenia. Schizophrenia Res 2015;162(1):294-5.

[51] Gagné AM, Paccalet T, Jomphe V, Lussier D, Maziade M. Electroertinographic response in youths at genetic risk of Schizophrenia and Bipolar Disorder and in normal controls: transversal and longitudinal differences and implication for the risk trajectory. Schizophr Bull 2018;44(suppl. 1):124.

[52] Maziade M, Paccalet T, Gagné AM, et al. Electroretinographic anomalies seen in patients affected by Schizophrenia or Bipolar Disorder are detectable early in children born to an affected parent: implications for the staging of risk status in childhood-adolescence. Schizophr Bull 2018;44(suppl. 1):11.

[53] Silverstein S, Demmin D, Erickson M, Thompson J, Paterno D. Netser R.v7.1 Electroretinographic anomalies in Schizophrenia and their relationships with retinal structure, visual functions, clinical symptoms, and medical comorbidities. Schizophr Bull 2018;44(suppl. 1):10-1.

[54] Laprevote V, Bernardin F, Schwitzer T, Schwan R. Electroretinogram anomalies in Schizophrenia patients with visual hallucinations. Schizophr Bull 2018;44 (suppl. 1):169.

[55] Demmin DL, Davis Q Roché M, Silverstein SM. Electroretinographic anomalies in schizophrenia. J Abnorm Psychol 2018;127(4):417.

[56] Pieraccini E, Bossini L, Martinucci M, et al. Assessment of photosensitivity through electroretinogram in patients with panic disorder (PD) and relationship with dopaminergic function. Eur Neuropsychopharmacol 2005;15(suppl. 3):5434.

[57] Bossini L, Padula L, Valdagno M, Castrogiovanni P. Electroretinogram B wave amplitude, dopaminergic system and panic disorder. Eur Neuropsychopharmacol 2008;18(suppl. 4):501.
[58] Nasser JA, Parigi AD, Merhige K, Wolper C, Geliebter A, Hashim SA. Electroretinographic detection of human brain dopamine response to oral food stimulation. Obesity 2013;21(5):976-80.

[59] Moschos MM, Moustafa GA, Gonidakis F, Papageorgiou C. Retinal and choroidal alterations in patients with anorexia nervosa without vision loss. Int J Eat Disord 2016;49(4):386-90.

[60] Bubl E, Werner L, Liang Y, et al. Evaluating the neurobiological correlates and impact of treatment on cognitive dysfunction in ADHD and Schizophrenia by means of the pattern electroretinogram. Schizophr Bull 2018;44(suppl. 1): 11 .

[61] Filip V, Balik J. Possible indication of dopaminergic blockade in man by electroretinography. Int Pharmacopsychiatry 1978;13:151-6.

[62] Fornaro M, Bandini F, Cestari L, et al. Electroretinographic modifications induced by agomelatine: a novel avenue to the understanding of the claimed antidepressant effect of the drug? Neuropsychiatr Dis Treat 2014;10:907.

[63] Perossini M, Fornaro P. Electroretinographic effects induced in humans by psychopharmacologic agents. Doc Ophthalmol 1990;75(1):1-6.

[64] Holopigian K, Clewner L, Seiple W, Kupersmith MJ. The effects of dopamine blockade on the human flash electroretinogram. Doc Ophthalmol 1994;86 (1):1-10.

[65] Fornaro P, Perossini M, Placidi GF, Dell'Osso L, Tassi G, Castrogiovanni P. Electroretinography (ERG) as a tool of investigation on dopaminergic activity in man. Res Comm Psychol Psychiatr Behav 1984;9(3):307-23.

[66] Morgane PJ, Galler JR, Mokler DJ. A review of systems and networks of the limbic forebrain/limbic midbrain. Prog Neurobiol 2005;75(2):143-60. 